\title{
Feasibility Evaluation of Function obtained by Product Combination
}

\author{
Yoko Nishihara, Junya Hibino, Junichi Fukumoto, and Ryosuke Yamanishi
}

\begin{abstract}
New products are frequently launched in the world. Most of new products can be assumed as combinations of existing products. All of the combinations do not always become seeds of new selling products. Each existing product has its own functions. A new function may be thought up by combining two existing products. If a thought up function is feasible, the combination may be a seed of a new selling product. This paper proposes a feasibility evaluation system of functions obtained by product combination for supporting idea creation of a new product. The system makes phrases representing new functions obtained by product combination. The system evaluates whether each of new functions is feasible or not. The system outputs combinations with feasible functions. The experiments for idea generation showed the following two findings: (1). Ideas of high novelty were obtained from combinations with functions of low feasibility. (2). Ideas of high utility were obtained from combinations with two new feasible functions.
\end{abstract}

Keywords: Feasible Function by Product Combination, Idea Generation Support, Novelty, Utility and Feasibility of Generated Idea

\section{Introduction}

New products are frequently launched in the world. Product developers always tries to know customer needs to create new and required products. Most of new products are approximate to combinations of existing products. For example, a mobile phone with a digital camera is approximate to a combination of a mobile phone and a digital camera. The mobile phone with a digital camera is much expensive, but has been bought and used by many customers.

All combinations of existing products do not always become seeds of new selling products. Let us assume that a new product is developed by combining a washing machine with a mobile phone. The new product has a function to remote-control a washing machine by a mobile phone. Though the new product is exactly developed from the combination of a washing machine and a mobile phone, most of customers would not buy the new product; because, the function, that is remote-control, is not novel and not useful for customers. In order to make a new product that has a potential to be a selling product, a combination for a new product must be satisfied with certain conditions to obtaining functions.

A mobile phone with a digital camera became a selling product in no time after launch. We suppose that a new product may become a selling product if a new available function 
obtained by product combination is realized. A mobile phone has a function to "send an e-mail." A digital camera has a function to "take a photo." If the two functions are used continuously, a new function to "take a photo and send an e-mail with a photo" is realized. The new function to "take a photo and send an e-mail with a photo" has been actually realized and spread among high school girls who enjoyed taking their photos and sending the photos to their friends. In this case, though developers just combined two functions of existing products, a new and attractive function is realized. We supposed that if a new function is realized by combining two existing products, many customers are attracted by a new product and will use the new product frequently.

This paper proposes a feasibility evaluation system of functions by product combination for supporting idea creation of new products. The system evaluates feasibility of functions obtained by product combination. The system outputs product combinations and the feasible functions. The system users refer the data and think up ideas of new products.

The proposed system describes a function of an existing product as a phrase of a verb and objects. A new function is also described as a phrase that is obtained by exchanging two objects between two phrases of existing product functions. The feasibility of a new function is evaluated by using a Web search engine.

\subsection{Previous Work of Idea Generation Support}

Brainstorming is one of the most famous methods to support idea generation [1]. Brainstorming can support conversations in idea generation. In the conversations, people think up ideas and express them to others after another. People are forbidden to deny ideas in the conversations. They can only accept ideas, and focus on good points of ideas to think up other new ideas. As other conversation support methods, Six Thinking Hat method [2] and problem criticizing method [3] have also been proposed. Though the previous researches support conversations in idea generation, the proposed system does not support conversations in idea creation. The proposed system supports to make idea seeds as subjects of conversations for idea generation.

To make idea seeds, KJ method is available and useful [4]. KJ method is one of the data classification methods. Users of $\mathrm{KJ}$ method prepare postcard-size cards in that users write data. One data is written in one card. Then, the users classify the cards with similar data into groups. Each of groups is given a label representing data. Next, the users integrate groups into big groups and give other labels to the big groups. Finally, the users obtain a classified data set with labels. The users refer the classified data set to make idea seeds. For data classification, Mind map is also useful [5]. The proposed system does not classify a large amount of data to support making idea seeds, but extract data from a large amount of data. The extracted data is about existing products that can make new products and make feasible functions by combining with other existing products.

A previous system can extract product combinations that may become seeds of new products for selling products [6]. The system supposes that a selling product satisfies with the following three conditions: 1. A product is the latest launched. 2. The other product is the conventional. 3. A combination of the two products is rare. The previous system does not evaluate feasibility of functions obtained by product combination. Each of products has functions that are used by customers. If obtained functions are not new for customers, most of customers do not try to use the new products because the new products may not be attractive for the customers. The proposed system evaluates whether each of obtained functions is feasible or not, and outputs combinations with feasible functions. The evaluated 


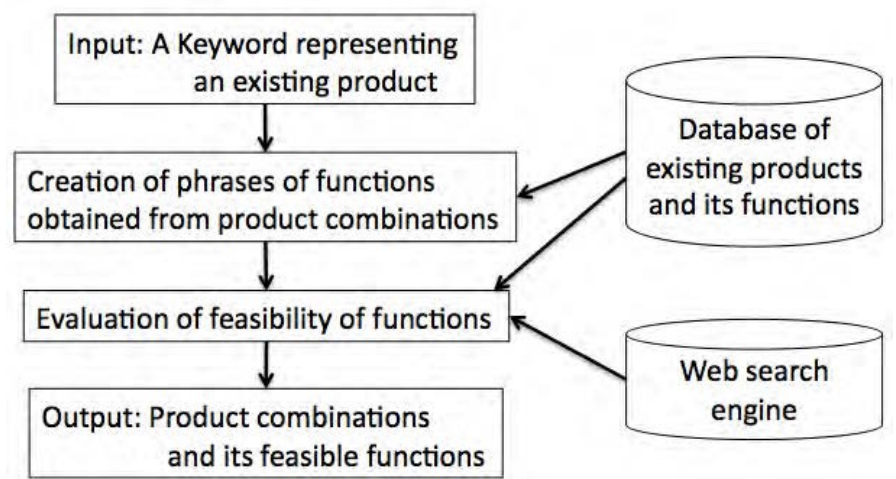

Figure 1: Flowchart of the proposed system.

functions support users to think up ideas of new products.

In thinking up ideas of new products, requirements from customers should be considered. However, even if a new function required by customers is added to a new product, the new product will not often become an attractive product [7]. To think up an idea for a new selling product, developers must find out essence of a required function. The proposed system outputs feasible functions obtained by product combination. The system users can imagine scenes of using the new functions, and think up ideas of new products with the new functions. We consider that scene imaging supports users to think up attractive products.

\section{Proposed System for Thinking Up Ideas of New Products}

Figure 1 shows a flowchart of the proposed system. A system user inputs a keyword representing an existing product. The system composes phrases representing new functions obtained from combinations of the input keyword and words representing existing products in a database. The system conducts search on the Web with an each of phrases as a query, and obtains the number of hits for each of phrases. If the number of hits for a phrase is more than one, the system evaluates that a new function represented by the phrase is feasible. The system finally outputs combinations and their feasible and new functions. A system user refers the outputs and imagines scenes in that new functions are used, in order to think up ideas of new products.

\subsection{Database of Existing Products and Available Functions}

The proposed system composes new functions by combining functions of existing products. It is difficult to automatically obtain function data of existing products. We, thus, prepared a database for words of existing products and phrases of available functions.

A function of an existing product is described as "(Action Word) (Object Words)." Each function has only one Action Word though some of functions have several Object Words. Table 1 shows examples of words of existing products and phrases of available functions described in the database. The database has 90 words of existing products, 162 functions of existing products (one product has 1.8 functions on average). 
Table 1: Database for words of existing products and phrases of available functions. A part of descriptions in a database is shown.

\begin{tabular}{|l|l|}
\hline Existing product & Available functions of an existing product \\
\hline Notebook & Take a note, Write an idea, Write a summary \\
\hline Projector & Project a picture on a screen, Project a movie on a screen \\
\hline Television & Watch a TV program \\
\hline Map & Search land information \\
\hline Digital Camera & Take a picture, Take a movie \\
\hline Speaker & Listen to music, Listen to voice \\
\hline Blender & Chop up food, Blend foods \\
\hline Rice wash up machine & Wash up rice \\
\hline Vacuum cleaner & Vacuum up the dirt \\
\hline Card case & Carry with business cards \\
\hline Rest Area & $\begin{array}{l}\text { Put gas in a car at a rest area, Have a lunch at a rest area, } \\
\text { Buy an item at a rest area, Use a lavatory at a rest area }\end{array}$ \\
\hline Cafeteria & Have a cup of coffee, Have a lunch \\
\hline Tonometer & Have one's blood pressure \\
\hline Refrigerator & Keep food in low temperature \\
\hline
\end{tabular}

\subsection{Composition of Phrases representing New Functions obtained by Product Combination}

The system composes phrases representing new functions obtained by combining existing products. A phrase for a function is made of phrases representing functions of two existing products. There are two ways of phrase composition.

We firstly explain how to compose phrases from functions with the same object. Suppose that two products (product $\mathrm{A}$ and product $\mathrm{B}$ ) are given, and each of function phrases is given as "(Action Word_A)(Object Word_A)" and "(Action Word_B $)($ Object Word_B $)$," respectively. New phrases are obtained by exchanging two Object Words, i.e., (Object Word $A$ ) and (Object Word_B). If the two Object Words are the same, i.e., (Object Word $A$ ) is equal to (Object Word_B), two phrases of new functions are represented as "(Action Word_A)(Object Word_A)" and "(Action Word_B)(OObject Word_B)." That is, the original function phrases are obtained again. For example, a function of a bicycle is given as "transfer a person fast", and a function of a fax machine is given as "contact a person." In this case, two Object Words ("a person" and "a person") are the same. Therefore, after exchanging Object Words, the same two function phrases are obtained.

Next, we explain how to compose phrases from functions with different objects. Suppose that two products (product A and product B) are given, and each of function phrases is given as "(Action Word_A)(Object Word_A)" and "(Action Word_B $)($ Object Word_B $)$," respectively. If the two Object Words are different from each other, two phrases of new functions are represented as "(Action Word_A $)($ Object Word_B $)$ " and "(Action Word_B $)($ Object Word $A$ )." For example, a function of a personal computer is given as "draw a picture," and a function of a fragrance is given as "put fragrance into food." In this case, two Object Words can be exchanged, and two new phrases "put fragrance into a picture" and "draw food" are obtained. 
Table 2: Groups for new functions obtained by product combination. Each of functions is classified by the level of feasibility.

\begin{tabular}{|l|l|}
\hline Group & Description of group \\
\hline Same object group & $\begin{array}{l}\text { Obtained phrases exist in the database of the proposed system. } \\
\text { Functions in this group are the most feasible. }\end{array}$ \\
\hline Big group & The number of hits for a phrase is more than 1,000,001. \\
\hline Middle group & $\begin{array}{l}\text { The number of hits for a phrase is more than 100,001 and less } \\
\text { than } 1,000,000 .\end{array}$ \\
\hline Small group & $\begin{array}{l}\text { The number of hits for a phrase is more than 11 and less than } \\
100,000 .\end{array}$ \\
\hline Very small group & $\begin{array}{l}\text { The number of hits for a phrase more than 1 and less than } 10 . \\
\text { Functions in this group are the least feasible. }\end{array}$ \\
\hline
\end{tabular}

Some phrases of functions have more than two Object Words. For the phrases with more than two Object Words, only the first Object Word is exchanged between two phrases of functions.

\subsection{Evaluation of Feasibility of New Functions represented by Phrases}

The proposed system evaluates the feasibility of new functions represented by phrases. We firstly explain how to evaluate the feasibility of new functions made of functions with the same object. If two phrases of new functions are made of functions with the same Object Words, two phrases exactly exist in the database shown in Table 1 . Therefore, the proposed system evaluates the two new functions as feasible.

Next, we explain how to evaluate the feasibility of new functions made of functions with different objects. If two phrases of new functions are made of functions with different Object Words, two phrases may not exist in the database. The proposed system uses a Web search engine to evaluate the feasibility of the two new functions. The system inputs each of phrases as a query into a Web search engine. If the number of hits for the query is more than one, the system evaluates a new function as feasible. A query is enclosed with double quotations to search Web pages including an exact query.

\subsection{Classification of Functions by Feasibility}

Each of functions represented by a phrase has each of feasibility levels. It is considered that the level of feasibility influences the characteristic of a generated idea. Therefore, the proposed system classifies new functions into five groups with the levels of feasibility.

The five groups are shown in Table 2. Each of groups is different in the number of hits for a phrase. "Same object group" is for functions made of functions with the same object. The other four groups, "Big group," "Middle group," "Small group," and "Very small group" are for functions made of functions with different objects. The four groups are referred to as "Different object group." We suppose that functions in "Same object group" are the most feasible, and functions in "Very small group" are the least. The ranges of the number of hits for each group were decided by referring Web pages [8, 9]. 
Table 3: Examples of outputs by the proposed system. Input keyword is "digital camera." Obtained functions, existing products for combinations, and groups of functions are shown. The numbers of hits are also shown in the most left-side column.

\begin{tabular}{|l|l|l|}
\hline Obtained function & Products for combination & Function group \\
\hline Take a photo \& Look a photo & Walkman & Same object \\
\hline Measure a photo & Distance meter & Big $(4,770,000)$ \\
\hline Bring with a photo & Card case & Middle $(395,000)$ \\
\hline Edit a movie & Personal computer & Small $(68,700)$ \\
\hline Measure a movie & Compass & Very small (3) \\
\hline
\end{tabular}

\subsection{Output: Combinations of Existing Products with New Feasible Functions}

The proposed system outputs combinations of existing products with new feasible functions. Output combinations are made of an input keyword and words representing other existing products. Functions are also output as phrases.

If functions are made of functions with the same object, phrases are output as a form "(Action Word_A) (Object Word_A) \& (Action Word_B) (Object Word_B)." The form implicates that both of two functions are feasible for one Object Word, and that the two functions can be used continuously. The order of using the functions is not mattered.

If functions are made of functions with different objects, each phrase is output as a form "(Action Word_A) (Object Word_B)" or "(Action Word_B) $($ Object Word_A)" with their groups.

Examples of system outputs are shown in Table 3. System users imagine scenes in that functions are used, and create ideas of new products by using output combinations.

\section{Evaluation Experiment}

We evaluated each of thought up ideas with the proposed system. Firstly, ideas obtained from "Same object group" were evaluated. Secondly, ideas obtained from "Different object group" were evaluated. Finally, all ideas were evaluated totally.

\subsection{Experimental Procedures}

Participants referred system outputs, and thought up ideas of new products. After idea generation, evaluators evaluated thought up ideas. The participants were 15 undergraduate students belonging to College of Information Science and Engineering. An experimenter (one of the authors) extracted three combinations from each of five groups shown in Table 2 , and showed a sheet in that 15 combinations ( $=3$ combinations $\times 5$ groups) were written. The experimenter made three sheets including different combinations respectively. An example of the sheet is shown in Table 4. Combinations in the sheets were not ordered in the level of feasibility.

The experimenter asked the participants as follows: 1. Imagine a scene in that a new function written in a sheet is used. 2. Think up an idea of a new product by combining two products written in the sheet. 3. Write a description of the thought up idea in the sheet. The experimenter did not limit the number of words used for writing descriptions. Each of 
Table 4: Example of a sheet for answering thought up ideas. Feasible new functions and product combinations for thinking up ideas of new products are shown.

\begin{tabular}{|l|l|l|}
\hline Feasible function(s) & Product A & Product B \\
\hline Put fragrance into a picture & Personal computer & Fragrance \\
\hline Browse food & Blender & Smart phone \\
\hline Play with a bread & Toaster oven & Robot \\
\hline $\begin{array}{l}\text { Project a picture on a screen \& Edit a } \\
\text { picture }\end{array}$ & Projector & Personal Computer \\
\hline Chop up food & Vacuum cleaner & Blender \\
\hline Keep food & Convenience store & Tank \\
\hline Write an e-mail & Smart phone & Robot \\
\hline Print out time & Wristwatch & Printer \\
\hline Look rice & Rice cooker & Television \\
\hline Wash a wrist & Wristwatch & Rice wash up machine \\
\hline Record the Internet & Smart phone & Recorder \\
\hline $\begin{array}{l}\text { Transfer a person fast \& Contact with } \\
\text { a person }\end{array}$ & Bicycle & Fax machine \\
\hline Enjoy radio communication & WiMAX & Music \\
\hline Take food & Blender & Memo \\
\hline $\begin{array}{l}\text { Contact with a person \& Transfer a } \\
\text { person fast }\end{array}$ & Smart phone & Bicycle \\
\hline
\end{tabular}

participants was asked to take more than 40 minutes for thinking up 15 ideas. In total, 225 ideas ( $=15$ ideas $\times 15$ participants) were thought up.

The evaluators were three undergraduate students belonging to College of Information Science and Engineering. Each of the evaluators read descriptions of ideas, and evaluated ideas. Evaluation scales were novelty, utility, and feasibility of an idea. The scales were decided by referring a previous study [10]. Each of ideas was evaluated by five-point rate (0: lowest and 4: highest). The experimenter averaged all rates for each scale.

We verified the following three hypotheses, (H1), (H2) and (H3). (H1): If the level of feasibility is low, a new function is rare for customers. By using a combination with such a new function, an idea of high novelty will be obtained. (H2): If the level of feasibility is high, a new function is familiar to customers. By using a combination with such a new function, an idea of high feasibility will be obtained. (H3): If both of two new functions are feasible, an idea of high utility will be obtained.

\subsection{Experimental Results}

Examples of thought up ideas are shown in Table 5. The averaged time was 49 minutes for thinking up 15 ideas. Each of ideas included a new function (new functions) and combinations of existing products.

Averaged rates of novelty, utility, and feasibility are shown in Table 6. The evaluation rate of the center was two because evaluation was conducted by five-point rate. For ideas obtained from "Same object group," the averaged rate of utility was 3.1 and the averaged rate of feasibility was 3.2 , which were higher than the center rate. In contrast, the averaged 
Table 5: Examples of thought up ideas obtained in the evaluation experiment.

Group
Thought up idea
(Feasible new function(s), combined products)
Same object group
A sofa that moves its head according to user's mood. It also gives an user massage.
(Care for a person\& make a person at home, Robot, Sofa)
Big group
A robot that reads sentences input by an user with a smart phone, and writes an e-mail
in handwriting.
(Write a e-mail, Smart phone, Robot)
Middle group
A print out service. An user holds a wristwatch over a printer. A printer prints out
time.
(Print out time, Wristwatch, Printer)
Small group
A toaster oven that prints a character's face on a bread until it is brown, and speaks in
a character's voice to children. Children are urged to eat breads fast.
(Play with a bread, Toaster oven, Robot)
Very small group
A washing machine that sorts clothes according to data input in personal computer.
For mother, for father, and for me. Socks and trousers.
(Edit clothes, Washing machine, Personal computer)

rate of novelty was 2.3 that was near to the center rate.

For ideas obtained from "Different object group," novel ideas were obtained from "Very small group" and "Middle group." Ideas of high utility were obtained from "Small group." The averaged rates of feasibility were between 2.9 to 3.1 , which were higher than the center rate. There were not big differences of feasibility among ideas of each group.

\section{Discussion}

We examine the evaluations for ideas thought up in the experiment.

\subsection{Examination of Evaluations for Ideas from "Same Object Group"}

The rates of utility and feasibility for ideas from "Same object group" were 3.1 and 3.2, respectively, which were higher than the center rate. In Table 5, an idea is shown, which was thought up by combining a robot with a sofa. The combination realized a new function to "care for a person \& make a person at home." The new function lead to make a new sofa that moves its head according to mood of an user. The sofa also gives massage to an user. If the idea is realized, a developed new product will support both of care workers and patients. Such an idea has high utility. The idea also had high feasibility because a new function was made of two existing functions. 
Table 6: Averaged evaluation rates of novelty, utility, and feasibility of thought up ideas.

\begin{tabular}{|l|l|l|l|}
\hline Group & Novelty & Utility & Feasibility \\
\hline Same Object & 2.3 & 3.1 & 3.2 \\
\hline Big & 2.5 & 2.4 & 3.0 \\
\hline Middle & 2.8 & 2.5 & 3.0 \\
\hline Small & 2.7 & 3.0 & 3.1 \\
\hline Very small & 2.8 & 2.8 & 2.9 \\
\hline
\end{tabular}

The rate of novelty was 2.3 that was near to the center rate. The participants thought up ideas by imagining scenes in that two existing functions were used. The scenes may be not new for the evaluators. Therefore, the rate of novelty was not high.

\subsection{Examination of Evaluations for Ideas from "Different Object Group"}

We examined the evaluations given to ideas from "Different object group." Novel ideas were obtained from "Very small group" and "Middle group." Table 5 shows an idea thought up by combining a washing machine with a personal computer, which function was classified into "Very small group." A new function to "edit clothes" is made by combining the two products. A participant thought up a new washing machine that sorts clothes according to data input in a personal computer. An idea about the new washing machine was novel. The novel idea was thought up because an obtained new function was rare.

Ideas of high utility were obtained from "Small group." Table 5 shows an idea that was obtained by combining a toaster oven with a robot. A new function to "play with a bread" is made by combining the two products. A participant thought up a new toaster oven that printed a character's face on a bread, and spoke in a character's voice to children. A product described in the idea may be useful especially for mothers who need to feed children breakfasts quickly in busy morning. The number of hits for a function phrase was not high. The low number means that the new function exist but is not seen frequently in the world. Evaluators rated high points to the idea because the rare function is realized in the idea.

Though the rates of feasibility were not different among groups. The rates were near to three though the center rate was two. It means that if a new function exist, the feasibility of an idea becomes high.

\subsection{Examination of Influences of Groups for Idea Generation}

Ideas of the highest novelty were obtained from "Very small group" and "Middle group." Those groups are for functions with the lower numbers of hits. If the number of hits is low, a function is low feasible but rare in the world. Ideas obtained from those two groups included low feasible but rare functions. That is the reason why ideas obtained from those groups were evaluated as novel. The hypothesis H1 was confirmed.

There are not much differences among groups in the evaluation of feasibility. The hypothesis $\mathrm{H} 2$ was not confirmed.

Ideas of the highest utility were obtained from "Same object group." Ideas from "Same object group" have two functions. Both of the functions exactly exist, and can be used con- 
tinuously. That is the reason why ideas obtained from "Same object group" were evaluated as with high utility. The hypothesis $\mathrm{H} 3$ was confirmed.

\section{Conclusions}

This paper proposed the feasibility evaluation system of functions obtained by product combination for supporting idea generation of new products. The proposed system composes phrases representing new functions made of two functions of existing products. The feasibility of functions is evaluated by the proposed system. If a composed function is feasible, the proposed system outputs a product combination and its feasible new function. System users refer the combinations with functions to thought up ideas of new products.

We experimented with the proposed system and obtained two findings: (1). Ideas of high novelty were obtained from combinations with functions of low feasibility, and (2). Ideas of high utility were obtained from combinations with two new feasible functions.

Adding a module to evaluate the continuity of two new functions with different objects would be the future work. The effectiveness of the module would be examined.

\section{References}

[1] Osborn, Alex F.: Applied Imagination, Scribner, 1979.

[2] de Bono, Edward: Six Thinking Hats: An Essential Approach to Business Management, Little, Brown, \& Company, 1985.

[3] Nishihara, Yoko and Ohsawa, Yukio, Communication Analysis focusing Negative Utterances in Combinatorial Thinking Games, The Review of Socionetwork Strategies, 4, 2, pp.31-46, 2010.

[4] Kawakita, Jiro, The KJ method? a scientific approach to problem solving, Kawakita Research Institute, 1975.

[5] Buzan, Tony, and Buzan, Barry, The Mind Map Book, Pearson Education Ltd., 2010.

[6] Nishihara, Yoko, Sunayama, Wataru and Yachida, Masahiko, Creative Activity Support by Discovering Effective Combinations, Systems and Computers in Japan, 38, 12 pp.99$111,2007$.

[7] Magnusson, Peter R., Customer-Oriented Product Development - Experiments Involving Users in Service Innovation -, Dissertation for the Degree of Doctor of Philosophy, Ph.D. in Business Administration, Stockholm School of Economics, 2003.

[8] http://books.google.co.jp/books?id=t2wY2NJcwvYC\&printsec

$=$ frontcover $\& \mathrm{hl}=\mathrm{ja} \&$ source $=$ gbs_ge_summary $\_$$\&$ cad $=0 \# \mathrm{v}=$ onepage

$\& q \& \mathrm{f}=$ false $(27 \mathrm{th} / \mathrm{Jan} . / 2014$ accessed $)$

[9] http://www.daiko-kikaku.com/sem_key_word1.html (27th/Jan./2014 accessed)

[10] Liikkanen, Lassi A., Hamalainen, Matti M., Haggman, Anders, Bjorklund, Tua, Koskinen, Mikko P., Quantitative Evaluation of the Effectiveness of Idea Generation in the Wild, Human Centered Design, 6776, pp.120-129, 2011. 